\title{
Upcoming market catalysts in Q1 2019
}

Potential catalysts in the first quarter of 2019 include a US approval decision for iclaprim (developed by Motif Bio) for acute bacterial skin and skin structure infections (ABSSSI), as well as top-line results for ResVax (developed by Novavax) for respiratory syncytial virus (RSV) and NanoFlu (developed by Novavax) for seasonal influenza.

Motif Bio's iclaprim, an intravenously administered inhibitor of microbial dihydrofolate reductase (DHFR), targets Gram-positive bacteria and is currently under FDA priority review for ABSSSI with a Prescription Drug User Fee Act (PDUFA) date of 13 February 2019. The new drug application (NDA) for iclaprim, which is an optimized analogue of the approved drug trimethoprim, is supported by results from two identical pivotal studies, known as REVIVE-1 and REVIVE-2. In both trials, iclaprim achieved non-inferiority with a $10 \%$ margin to an active comparator, vancomycin, for both early clinical response (48-72 h after the start of administration) and clinical cure at 7-14 days after study drug discontinuation. In addition, in REVIVE-1 and REVIVE-2, 60.4\% and $54.6 \%$, respectively, of patients receiving iclaprim demonstrated resolution or near resolution at the end of therapy, compared with $58.3 \%$ and $55.4 \%$ of patients receiving vancomycin in an analysis of a pre-specified secondary end point. Having received qualified infectious disease product (QIDP) and fast track designation, iclaprim is likely to be approved for ABSSSI and will help fill the unmet need for novel antibiotics active against Gram-positive bacteria such as methicillin-resistant Staphylococcus aureus (MRSA).

Novavax anticipates prespecified interim efficacy analysis data from the phase III Prepare trial of ResVax, a recombinant nanoparticle RSV F vaccine, in the first quarter of 2019. This trial is pioneering a strategy of immunizing pregant women with the aim of protecting their children from RSV in the first few months of life.

In December 2017, Novavax conducted an informational analysis related to the prevention of medically significant RSV-positive lower respiratory tract infection in a subset of 1,300 infants from the Prepare trial. This analysis enabled Novavax to conclude that the vaccine's potential observed efficacy in this subset group is in the range $45-100 \%$. With the previous readout from the pivotal phase III Resolve trial in the elderly showing that an unadjuvanted formulation of ResVax did not meet the prespecified primary or secondary efficacy objectives, top-line results from the phase III Prepare trial, which uses an alum-adjuvanted formulation, will be of utmost importance to demonstrate the efficacy of ResVax. If approved for maternal immunization, Novavax could tap into an enormous RSV market as currently there are no approved RSV vaccines available, as evidenced by its fast track designation by the FDA.

Results from a phase II trial of NanoFlu in the elderly against the current standard of care (Fluzone HD) are also expected in the first quarter of 2019. NanoFlu is a nanoparticle-based seasonal influenza vaccine, formulated with or without Matrix M-1 adjuvant. As a cell-based recombinant vaccine, NanoFlu possesses a potential advantage over the current standard of care in the elderly population, the egg-based Fluzone HD, in that there is reduced potential for antigenic mutations during the manufacturing process that can lead to impaired protection against circulating strains. In a phase I/II study in older adults, NanoFlu demonstrated significantly higher haemagglutination inhibition (HAl) antibody responses against a panel of vaccine-homologous, and historically and forward-drifted, influenza virus strains than Fluzone HD. Although immunogenicity will not necessarily translate to increased protection in future studies, the data suggest that NanoFlu could hold a competitive profile in a saturated market segment.

$$
\begin{array}{r}
\text { Khang Hoang is at Sagient Research Systems, } \\
\text { an Informa business, San Diego, CA, USA. } \\
\text { e-mail: KHoang@sagientresearch.com } \\
\text { doi:10.1038/nrd.2018.223 } \\
\text { Published online 28 Dec 2018 }
\end{array}
$$

Competing interests

The author declares no competing interests. 